\title{
Darboux Transformation and Explicit Solutions for a Generalized Sawada-Kotera Equation
}

\author{
Guo-Liang $\mathrm{He}^{1}$ and Ting $\mathrm{Su}^{2}$ \\ ${ }^{1}$ Department of Mathematics and Information Science, Zhengzhou University of Light Industry, Zhengzhou 450002, China \\ ${ }^{2}$ Department of Mathematics, Henan Institute of Engineering, Zhengzhou 451191, China \\ Correspondence should be addressed to Guo-Liang He; glhemath@163.com
}

Received 17 April 2013; Accepted 13 May 2013

Academic Editors: A. Bellouquid, M. Mei, J. Park, and F. Tadeo

Copyright (c) 2013 G.-L. He and T. Su. This is an open access article distributed under the Creative Commons Attribution License, which permits unrestricted use, distribution, and reproduction in any medium, provided the original work is properly cited.

\begin{abstract}
A generalized Sawada-Kotera equation and its Lax pairs are proposed. With the help of the gauge transformation between spectral problems, a Darboux transformation for the generalized SK equation is constructed. As an application of the Darboux transformation, we give some explicit solutions of the generalized SK equation such as the rational solutions, soliton solutions, and periodic solutions.
\end{abstract}

\section{Introduction}

The Sawada-Kotera (SK) equation

$$
u_{t}=-u_{x x x x x}+15\left(u u_{x x}-u^{3}\right)_{x}
$$

was first proposed by Sawada and Kotera when they gave a method for finding $\mathrm{N}$-soliton solutions of the $\mathrm{KdV}$ equation and the KdV-like equation [1]. In [2], Caudrey et al. showed that (1) was a member of a new hierarchy of KdV equations. The SK equation's physical importance was illustrated by Aiyer et al. in [3]. Then, the equation has been investigated by many authors [4-8]. The aim of the present paper is using the Darboux transformation [9-12] to study a generalized SK equation:

$$
\begin{aligned}
& u_{t}=-u_{x x x x x}+15\left(u u_{x x}-u^{3}\right)_{x}-15\left(v u_{x}\right)_{x}-10 v v_{x}, \\
& v_{t}=-v_{x x x x x}+15\left(u v_{x x}+v v_{x}-3 v u^{2}\right)_{x}+30\left(v u_{x}\right)_{x x} .
\end{aligned}
$$

The present paper is organized as follows. In Section 2, with the aid of the Lax pairs of the SK equation $[13,14]$ and extending them by adding one potential function, we propose a generalized SK equation and its Lax pairs. Based on the gauge transformation between spectral problems, we derive a Darboux transformation of the generalized SK equation. In Section 3, the Darboux transformation is applied to the generalized SK equation, by which explicit solutions (we have verified the correctness of the solutions by using the Mathematic 5.0.) of the generalized SK equation are derived, including rational solutions, soliton solutions, and periodic solutions.

\section{Darboux Transformation of the Generalized Sawada-Kotera Equation}

In this section, we will derive a generalized SK equation and its Darboux transformation. To this end, we first introduce the Lax pairs:

$$
\mathscr{L} \psi=\lambda \psi, \quad \psi_{t}=\mathscr{B} \psi,
$$

where operators $\mathscr{L}$ and $\mathscr{B}$ are defined as follows:

$$
\begin{gathered}
\mathscr{L}=\partial^{3}-3 u \partial+v \\
\mathscr{B}=9 \partial^{5}-45 u \partial^{3}+15\left(v-3 u_{x}\right) \partial^{2} \\
+15\left(3 u^{2}-2 u_{x x}+v_{x}\right) \partial+10\left(v_{x x}-3 u v\right) .
\end{gathered}
$$

Then the compatibility condition between the two equations of (3) yields the Lax equation, $\mathscr{L}_{t}=[\mathscr{B}, \mathscr{L}]$, which is equivalent to the generalized SK equation:

$$
\begin{aligned}
& u_{t}=-u_{x x x x x}+15\left(u u_{x x}-u^{3}\right)_{x}-15\left(v u_{x}\right)_{x}-10 v v_{x}, \\
& v_{t}=-v_{x x x x x}+15\left(u v_{x x}+v v_{x}-3 u^{2} v\right)_{x}+30\left(u_{x} v\right)_{x x} .
\end{aligned}
$$


If we choose $v=0$ and $v=-(3 / 2) u_{x},(5)$ can be, respectively, reduced to the SK equation:

$$
u_{t}=-u_{x x x x x}-45 u^{2} u_{x}+15 u u_{x x x}+15 u_{x} u_{x x}
$$

and the Kaup-Kupershmidt equation:

$$
u_{t}=-u_{x x x x x}-45 u^{2} u_{x}+15 u u_{x x x}+\frac{75}{2} u_{x} u_{x x} .
$$

Theorem 1. Let $f$ satisfy (3) with $\lambda=\lambda_{0}$ and $A=-(\ln f)_{x}$. Then the following Darboux transformation gives the relation about the original solutions $u, v$ of (5) and its new ones $\bar{u}, \bar{v}$ :

$$
\begin{gathered}
\bar{u}=u+A_{x}, \\
\bar{v}=v-3 u_{x}+3 A A_{x}-3 A_{x x} .
\end{gathered}
$$

Proof. Assume that $\psi$ satisfies (3) and $A=-(\ln f)_{x}$. Let

$$
\bar{\psi}=\psi_{x}+A \psi .
$$

Using the first expression of (3), a direct calculation gives the following equations:

$$
\begin{aligned}
\bar{\psi}_{x}=\psi_{x x}+A \psi_{x}+A_{x} \psi, \\
\bar{\psi}_{x x}=A \psi_{x x}+\left(3 u+2 A_{x}\right) \psi_{x}+\left(A_{x x}-v+\lambda\right) \psi, \\
\bar{\psi}_{x x x}=3\left(u+A_{x}\right) \psi_{x x}+\left(3 u_{x}-v+3 A_{x x}+3 u A+\lambda\right) \psi_{x} \\
\quad+\left(A_{x x x}-v_{x}-v A+\lambda A\right) \psi .
\end{aligned}
$$

Substituting (9) and (10) into the following equation:

$$
\overline{\mathscr{L}} \bar{\psi}=\lambda \bar{\psi},
$$

where

$$
\overline{\mathscr{L}}=\partial^{3}-3 \bar{u} \partial+\bar{v}
$$

and comparing the coefficients of $\psi, \psi_{x}$, and $\psi_{x x}$, we obtain the following:

$$
\begin{gathered}
\bar{u}-u-A_{x}=0, \\
3 \bar{u} A-\bar{v}-3 u_{x}+v-3 A_{x x}-3 u A=0, \\
3 \bar{u} A_{x}-\bar{v} A-A_{x x x}+v_{x}+v A=0 .
\end{gathered}
$$

Equation (13) implies the following:

$$
\begin{gathered}
\bar{u}=u+A_{x}, \\
\bar{v}=v-3 u_{x}+3 A A_{x}-3 A_{x x} .
\end{gathered}
$$

Substituting (15) into (14) and integrating it once, we have the following:

$$
3 u A+3 A A_{x}-A^{3}-A_{x x}+v=\lambda_{0},
$$

where $\lambda_{0}$ is a constant of integration. Through direct calculations, we arrive at the following:

$$
\begin{aligned}
& -\frac{f_{x x}}{f}=A_{x}-A^{2}, \\
& -\frac{f_{x x x}}{f}=A_{x x}-3 A A_{x}+A^{3},
\end{aligned}
$$

$$
\begin{aligned}
-\frac{f_{4 x}}{f}= & A_{x x x}-4 A A_{x x}-3 A_{x}^{2}+6 A^{2} A_{x}-A^{4}, \\
-\frac{f_{5 x}}{f}= & A_{x x x x}-5 A A_{x x x}-10 A_{x} A_{x x} \\
& +15 A A_{x}^{2}+10 A^{2} A_{x x}-10 A^{3} A_{x}+A^{5} .
\end{aligned}
$$

Using (17) and $A=-(\ln f)_{x}$, a simple reduction shows that (16) gives rise to the following:

$$
\mathscr{L} f=\lambda_{0} f .
$$

Similarly, we consider the following equation:

$$
\bar{\psi}_{t}=\overline{\mathscr{B}} \bar{\psi},
$$

where

$$
\begin{aligned}
\overline{\mathscr{B}}= & 9 \partial^{5}-45 \bar{u} \partial^{3}+15\left(\bar{v}-3 \bar{u}_{x}\right) \partial^{2} \\
& +15\left(3 \bar{u}^{2}-2 \bar{u}_{x x}+\bar{v}_{x}\right) \partial+10\left(\bar{v}_{x x}-3 \overline{u v}\right) .
\end{aligned}
$$

Seeing (3), (8), and (9), a direct calculation shows that (19) gives the following:

$$
\begin{aligned}
A_{t}= & 9 A_{x x x x x}-10 v_{x x x}-45 u A_{x x x}+30 u v_{x} \\
& +15 v A_{x x}-90 A_{x x}^{2}+30 u_{x} v-90 u_{x} A_{x x} \\
& +45 u^{2} A_{x}+90 u A_{x}^{2}+90 A_{x}^{3}-75 u_{x x} A_{x} \\
& -120 A_{x} A_{x x x}+15 v_{x} A_{x}+270 A A_{x} A_{x x} \\
& +30 A^{2} A_{x x x}-30 A A_{x x x x}-30 u_{x x x} A \\
& -90 u A^{2} A_{x}+90 u A A_{x x}+90 u u_{x} A \\
& -30 v A A_{x}-90 A^{2} A_{x}^{2}+90 u_{x} A A_{x}
\end{aligned}
$$

which together with (17) implies the following:

$$
f_{t}=\mathscr{B} f .
$$

This means that both of the Lax pairs (3) and (11) and (19) have the same form; that is, they lead to the same equation (5). Therefore, original solutions $u, v$ of the generalized SK equation (5) are mapped into its new ones $\bar{u}, \bar{v}$ by the Darboux transformation (8).

\section{Explicit Solutions of the Generalized Sawada-Kotera Equation}

In this section, we will construct explicit solutions of the generalized SK equation (5) by using the Darboux transformation (8).

(I) We choose a trivial solution $u=0, v=0$ of (5). Then (3) with $\lambda=\lambda_{0}$ is reduced to the following:

$$
\psi_{x x x}=\lambda_{0} \psi, \quad \psi_{t}=9 \psi_{x x x x x} .
$$


Let $\lambda_{0}=-k^{3}(k \neq 0)$. We can see that $(23)$ has a general solution:

$$
\begin{aligned}
f= & c_{1} \exp \left(\Delta_{1}\right)+c_{2} \exp \left(-\frac{1}{2} \Delta_{1}\right) \cos \left(\Delta_{2}\right) \\
& +c_{3} \exp \left(-\frac{1}{2} \Delta_{1}\right) \sin \left(\Delta_{2}\right),
\end{aligned}
$$

where $c_{j},(j=1,2,3)$ are constants and

$$
\Delta_{1}=-k x-9 k^{5} t, \quad \Delta_{2}=\frac{\sqrt{3}}{2}\left(k x-9 k^{5} t\right) .
$$

Using the Darboux transformation (8), we get an explicit solution of (5)

$$
\begin{gathered}
\bar{u}=\frac{3\left(c_{2}^{2}+c_{3}^{2}\right) k^{2} \exp \left(-3 \Delta_{1}\right)-6 c_{1} k^{2} \exp \left(-(3 / 2) \Delta_{1}\right)\left[\left(c_{2}+\sqrt{3} c_{3}\right) \cos \left(\Delta_{2}\right)-\left(\sqrt{3} c_{2}-c_{3}\right) \sin \left(\Delta_{2}\right)\right]}{4\left[c_{1}+c_{2} \exp \left(-(3 / 2) \Delta_{1}\right) \cos \left(\Delta_{2}\right)+c_{3} \exp \left(-(3 / 2) \Delta_{1}\right) \sin \left(\Delta_{2}\right)\right]^{2}}, \\
\bar{v}=\left(-\left\{9 \operatorname { e x p } ( - \frac { 3 } { 2 } \Delta _ { 1 } ) k ^ { 3 } \left(4 c_{1}^{2}\left[\left(c_{2}-\sqrt{3} c_{3}\right) \cos \left(\Delta_{2}\right)+\left(\sqrt{3} c_{2}+c_{3}\right) \sin \left(\Delta_{2}\right)\right]\right.\right.\right. \\
+\exp \left(-3 \Delta_{1}\right)\left(\left[\left(c_{2}-\sqrt{3} c_{3}\right) \cos \left(\Delta_{2}\right)+\left(\sqrt{3} c_{2}+c_{3}\right) \sin \left(\Delta_{2}\right)\right]\left(c_{2}^{2}+c_{3}^{2}\right)\right) \\
+2 \exp \left(-\frac{3}{2} \Delta_{1}\right) c_{1}\left(\left(6+\cos \left(2 \Delta_{2}\right)-\sqrt{3} \sin \left(2 \Delta_{2}\right)\right) c_{2}^{2}\right. \\
+2\left(\sin \left(2 \Delta_{2}\right)+\sqrt{3} \cos \left(2 \Delta_{2}\right)\right) c_{2} c_{3} \\
\left.\left.\left.\left.+\left(6-\cos \left(2 \Delta_{2}\right)+\sqrt{3} \sin \left(2 \Delta_{2}\right)\right) c_{3}^{2}\right)\right)\right\}\right) \\
\times\left(8\left[c_{1}+c_{2} \exp \left(-\frac{3}{2} \Delta_{1}\right) \cos \left(\Delta_{2}\right)+c_{3} \exp \left(-\frac{3}{2} \Delta_{1}\right) \sin \left(\Delta_{2}\right)\right]^{3}\right) .
\end{gathered}
$$

Particularly, when we choose $c_{1}=0, c_{2}=c_{3}=1$, we can get a periodic solution of (5):

$$
\begin{gathered}
\bar{u}=\frac{3 k^{2}}{2\left[\cos \left(\Delta_{2}\right)+\sin \left(\Delta_{2}\right)\right]^{2}}, \\
\bar{v}=-3 k^{3}+3 k^{3}\left(\frac{1}{2}+\frac{\sqrt{3}}{2} \frac{\cos \left(\Delta_{2}\right)-\sin \left(\Delta_{2}\right)}{\cos \left(\Delta_{2}\right)+\sin \left(\Delta_{2}\right)}\right)^{3} \\
+3 k^{3} \frac{2 \sin \left(2 \Delta_{2}\right)-1}{\left[\cos \left(\Delta_{2}\right)+\sin \left(\Delta_{2}\right)\right]^{2}} .
\end{gathered}
$$

Plots of the solutions are given in Figures 1 and 2.

(II) We consider the trivial solution $u=0, v=1$ of (5). Then (3) with $\lambda=\lambda_{0}$ is reduced to the following:

$$
\psi_{x x x}=\left(\lambda_{0}-1\right) \psi, \quad \psi_{t}=9 \psi_{x x x x x}+15 \psi_{x x} .
$$

Case 1. When $\lambda_{0}=1$, it is easy to see that (28) has a general solution:

$$
f=\alpha x^{2}+\beta x+\gamma+30 \alpha t
$$

where $\alpha, \beta$, and $\gamma$ are constants. Using the Darboux transformation (8), we get a rational solution of the generalized SK equation (5):

$$
\begin{gathered}
\bar{u}=\frac{2 \alpha^{2} x^{2}+2 \alpha \beta x+\beta^{2}-2 \alpha \gamma-60 \alpha^{2} t}{\left[\alpha x^{2}+\beta x+\gamma+30 \alpha t\right]^{2}}, \\
\bar{v}=1+\frac{3\left(\beta^{2}-4 \alpha(\gamma+30 \alpha t)\right)(\beta+2 \alpha x)}{\left[\alpha x^{2}+\beta x+\gamma+30 \alpha t\right]^{3}} .
\end{gathered}
$$

Case 2. When $\lambda_{0}=1-k^{3}(k \neq 0)$, (28) has a general solution:

$$
\begin{aligned}
f= & c_{1} \exp \left(\Delta_{1}\right)+c_{2} \exp \left(-\frac{1}{2} \Delta_{1}\right) \cos \left(\Delta_{2}\right) \\
& +c_{3} \exp \left(-\frac{1}{2} \Delta_{1}\right) \sin \left(\Delta_{2}\right),
\end{aligned}
$$

where $c_{j},(j=1,2,3)$ are constants and

$$
\begin{gathered}
\Delta_{1}=-k x-\left(9 k^{5}-15 k^{2}\right) t, \\
\Delta_{2}=\frac{\sqrt{3}}{2}\left(k x-9 k^{5} t+15 k^{2} t\right) .
\end{gathered}
$$

Using the Darboux transformation (8), we get an explicit solution of (5): 


$$
\begin{gathered}
\bar{u}=\frac{3\left(c_{2}^{2}+c_{3}^{2}\right) k^{2} \exp \left(-3 \Delta_{1}\right)-6 c_{1} k^{2} \exp \left(-(3 / 2) \Delta_{1}\right)\left[\left(c_{2}+\sqrt{3} c_{3}\right) \cos \left(\Delta_{2}\right)-\left(\sqrt{3} c_{2}-c_{3}\right) \sin \left(\Delta_{2}\right)\right]}{4\left[c_{1}+c_{2} \exp \left(-(3 / 2) \Delta_{1}\right) \cos \left(\Delta_{2}\right)+c_{3} \exp \left(-(3 / 2) \Delta_{1}\right) \sin \left(\Delta_{2}\right)\right]^{2}}, \\
\bar{v}=\left(1-\left\{9 \operatorname { e x p } ( - \frac { 3 } { 2 } \Delta _ { 1 } ) k ^ { 3 } \left(4 c_{1}^{2}\left[\left(c_{2}-\sqrt{3} c_{3}\right) \cos \left(\Delta_{2}\right)+\left(\sqrt{3} c_{2}+c_{3}\right) \sin \left(\Delta_{2}\right)\right]\right.\right.\right. \\
+\exp \left(-3 \Delta_{1}\right)\left(\left[\left(c_{2}-\sqrt{3} c_{3}\right) \cos \left(\Delta_{2}\right)+\left(\sqrt{3} c_{2}+c_{3}\right) \sin \left(\Delta_{2}\right)\right]\left(c_{2}^{2}+c_{3}^{2}\right)\right) \\
+2 \exp \left(-\frac{3}{2} \Delta_{1}\right) c_{1}\left(\left(6+\cos \left(2 \Delta_{2}\right)-\sqrt{3} \sin \left(2 \Delta_{2}\right)\right) c_{2}^{2}\right. \\
+2\left(\sin \left(2 \Delta_{2}\right)+\sqrt{3} \cos \left(2 \Delta_{2}\right)\right) c_{2} c_{3} \\
\left.\left.\left.\left.+\left(6-\cos \left(2 \Delta_{2}\right)+\sqrt{3} \sin \left(2 \Delta_{2}\right)\right) c_{3}^{2}\right)\right)\right\}\right) \\
\times\left(8\left[c_{1}+c_{2} \exp \left(-\frac{3}{2} \Delta_{1}\right) \cos \left(\Delta_{2}\right) c_{3} \exp \left(-\frac{3}{2} \Delta_{1}\right) \sin \left(\Delta_{2}\right)\right]^{3}\right)^{-1} .
\end{gathered}
$$

(III) We choose another trivial solution $u=1, v=0$ of (5). Then (3) with $\lambda=\lambda_{0}$ is reduced to the following:

$$
\psi_{x x x}=3 \psi_{x}+\lambda_{0} \psi, \quad \psi_{t}=9 \psi_{x x x x x}-45 \psi_{x x x}+45 \psi_{x} .
$$

Case 1. For $\lambda_{0}=0$, a direct calculation gives a general solution of (34):

$$
f=c_{1} \exp (\Delta)+c_{2} \exp (-\Delta)
$$

where $c_{1}, c_{2}$ are constants and $\Delta=\sqrt{3} x-9 \sqrt{3} t$. Using the Darboux transformation (8), we get a soliton solution of the generalized SK equation (5) $\left(c_{1}=c_{2}=1\right)$ :

$$
\begin{gathered}
\bar{u}=3[\tanh (\Delta)]^{2}-2, \\
\bar{v}=9 \sqrt{3}[\tanh (\Delta)]^{3}-9 \sqrt{3} \tanh (\Delta) .
\end{gathered}
$$

Plots of the solutions are given in Figures 3 and 4.

Case 2. For $\lambda_{0}=k^{3}-3 k(k \neq 0)$, (34) has a general solution:

$$
f=c_{1} \exp \left(\Delta_{1}\right)+c_{2} \exp \left(\Delta_{2}\right)+c_{3} \exp \left(\Delta_{3}\right)
$$

where $c_{j},(j=1,2,3)$ are constants and

$$
\begin{aligned}
& \Delta_{1}= k x+\left(9 k^{5}-45 k^{3}+45 k\right) t \\
& \Delta_{2}= \frac{-k+\sqrt{12-3 k^{2}}}{2} x \\
&-\frac{9}{2}\left(5 k-5 k^{3}+k^{5}+\sqrt{12-3 k^{2}}\right. \\
& \Delta_{3}=\left.-3 k^{2} \sqrt{12-3 k^{2}}+k^{4} \sqrt{12-3 k^{2}}\right) t \\
&+\frac{9}{2}\left(-5 k+5 k^{3}-k^{5}+\sqrt{12-3 k^{2}}\right. \\
&\left.-3 k^{2} \sqrt{12-3 k^{2}}+k^{4} \sqrt{12-3 k^{2}}\right) t .
\end{aligned}
$$

Using the Darboux transformation (8), we get an explicit solution of (5):

$$
\begin{gathered}
\bar{u}=1+\frac{3}{2} \frac{2 c_{2} c_{3}\left(k^{2}-4\right) \exp \left(\Delta_{2}+\Delta_{3}\right)-c_{1} c_{2}\left(2+k^{2}-k \sqrt{12-3 k^{2}}\right) \exp \left(\Delta_{1}+\Delta_{2}\right)-c_{1} c_{3}\left(2+k^{2}+k \sqrt{12-3 k^{2}}\right) \exp \left(\Delta_{1}+\Delta_{3}\right)}{\left[c_{1} \exp \left(\Delta_{1}\right)+c_{2} \exp \left(\Delta_{2}\right)+c_{3} \exp \left(\Delta_{3}\right)\right]^{2}} \\
\bar{v}=3 k^{3}-9 k+3 \frac{\Delta}{\left[c_{1} \exp \left(\Delta_{1}\right)+c_{2} \exp \left(\Delta_{2}\right)+c_{3} \exp \left(\Delta_{3}\right)\right]^{3}},
\end{gathered}
$$




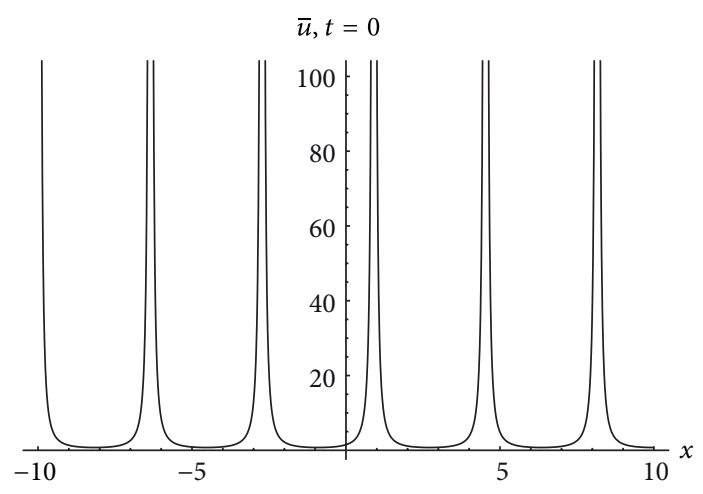

Figure 1: $\bar{u}$.

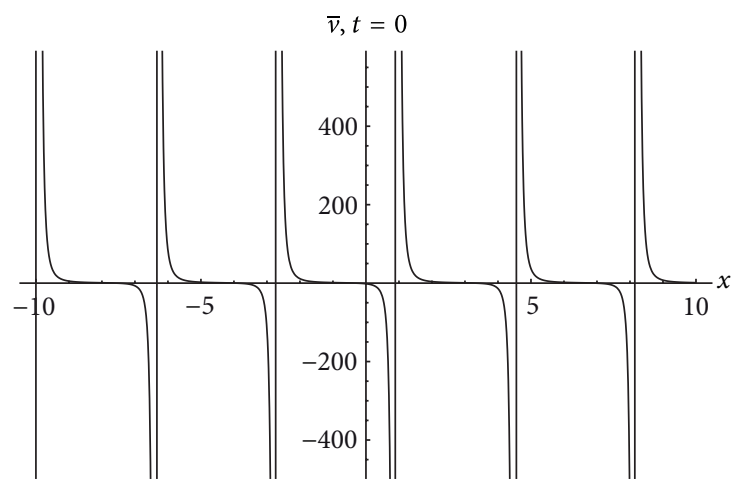

FIGURE 2: $\bar{v}$.

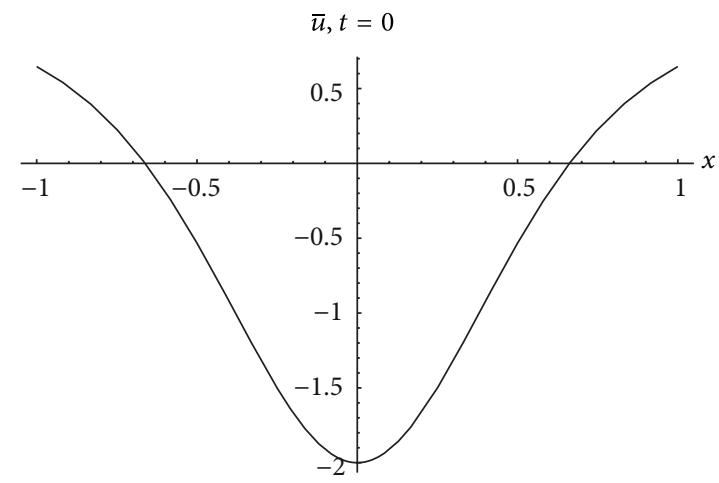

FIgURE 3: $\bar{u}$.

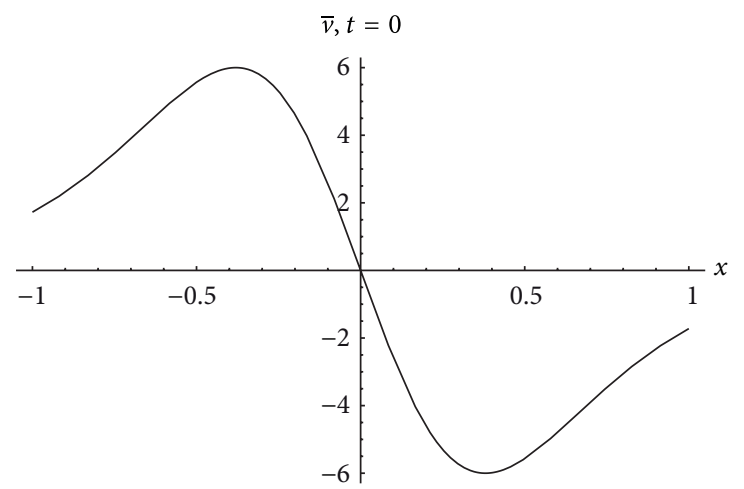

FIGURE 4: $\bar{v}$. 
where

$$
\begin{aligned}
\Delta= & k\left(3-k^{2}\right)\left[c_{1}^{3} \exp \left(3 \Delta_{1}\right)\right. \\
& +c_{2}^{3} \exp \left(3 \Delta_{2}\right)+c_{3}^{3} \exp \left(3 \Delta_{3}\right) \\
& \left.-12 c_{1} c_{2} c_{3} \exp \left(\Delta_{1}+\Delta_{2}+\Delta_{3}\right)\right] \\
+ & \frac{3}{2} c_{2}^{2} c_{3} \exp \left(2 \Delta_{2}+\Delta_{3}\right) \\
\times & {\left[2 k-k^{3}-4 \sqrt{12-3 k^{2}}+k^{2} \sqrt{12-3 k^{2}}\right] } \\
+ & \frac{3}{2} c_{2} c_{3}^{2} \exp \left(\Delta_{2}+2 \Delta_{3}\right) \\
\times & {\left[2 k-k^{3}+4 \sqrt{12-3 k^{2}}-k^{2} \sqrt{12-3 k^{2}}\right] } \\
+ & \frac{3}{2} c_{1} c_{2}^{2} \exp \left(\Delta_{1}+2 \Delta_{2}\right) k\left(8-k^{2}-k \sqrt{12-3 k^{2}}\right) \\
+ & \frac{3}{2} c_{1}^{2} c_{2} \exp \left(2 \Delta_{1}+\Delta_{2}\right)\left(1+k^{2}\right)\left(k-\sqrt{12-3 k^{2}}\right) \\
+ & \frac{3}{2} c_{1}^{2} c_{3} \exp \left(2 \Delta_{1}+\Delta_{3}\right)\left(1+k^{2}\right)\left(k+\sqrt{12-3 k^{2}}\right) \\
& 3 c_{3}^{2} \exp \left(\Delta_{1}+2 \Delta_{3}\right) k\left(8-k^{2}+k \sqrt{12-3 k^{2}}\right)
\end{aligned}
$$

[6] S. Y. Lou, "Twelve sets of symmetries of the Caudrey-DoddGibbon-Sawada-Kotera equation," Physics Letters A, vol. 175, no. 1, pp. 23-26, 1993.

[7] Y.-G. Xu, X.-W. Zhou, and L. Yao, "Solving the fifth order Caudrey-Dodd-Gibbon (CDG) equation using the exp-function method," Applied Mathematics and Computation, vol. 206, no. 1, pp. 70-73, 2008.

[8] A. H. Salas, "Exact solutions for the general fifth KdV equation by the exp function method," Applied Mathematics and Computation, vol. 205, no. 1, pp. 291-297, 2008.

[9] V. B. Matveev and M. A. Salle, Darboux Transformations and Solitons, Springer Series in Nonlinear Dynamics, Springer, Berlin, Germany, 1991.

[10] D. Levi, "On a new Darboux transformation for the construction of exact solutions of the Schrödinger equation," Inverse Problems, vol. 4, no. 1, pp. 165-172, 1988.

[11] C. H. Gu and Z. X. Zhou, "On Darboux transformations for soliton equations in high-dimensional spacetime," Letters in Mathematical Physics, vol. 32, no. 1, pp. 1-10, 1994.

[12] X. G. Geng and G. L. He, "Some new integrable nonlinear evolution equations and Darboux transformation," Journal of Mathematical Physics, vol. 51, no. 3, Article ID 033514, 21 pages, 2010.

[13] J. Satsuma and D. J. Kaup, "A Bäcklund transformation for a higher order Korteweg-de Vries equation," Journal of the Physical Society of Japan, vol. 43, no. 2, pp. 692-726, 1977.

[14] J. M. Dye and A. Parker, "On bidirectional fifth-order nonlinear evolution equations, Lax pairs, and directionally dependent solitary waves," Journal of Mathematical Physics, vol. 42, no. 6, pp. 2567-2589, 2001.

\section{Acknowledgments}

This work was supported by National Natural Science Foundation of China (no. 11171312) and Henan Natural Science Foundation of Basic Research (no. 102300410214).

\section{References}

[1] K. Sawada and T. Kotera, "A method for finding N-soliton solutions of the K.d.V. equation and K.d.V.-like equation," Progress of Theoretical Physics, vol. 51, pp. 1355-1367, 1974.

[2] P. J. Caudrey, R. K. Dodd, and J. D. Gibbon, "A new hierarchy of Korteweg-de Vries equations," Proceedings of the Royal Society London A, vol. 351, no. 1666, pp. 407-422, 1976.

[3] R. N. Aiyer, B. Fuchssteiner, and W. Oevel, "Solitons and discrete eigenfunctions of the recursion operator of nonlinear evolution equations. I. The Caudrey-Dodd-Gibbon-Sawada-Kotera equation," Journal of Physics A, vol. 19, no. 18, pp. 3755-3770, 1986.

[4] A.-M. Wazwaz, "Multiple-soliton solutions for the fifth order Caudrey-Dodd-Gibbon (CDG) equation," Applied Mathematics and Computation, vol. 197, no. 2, pp. 719-724, 2008.

[5] A.-M. Wazwaz, "Analytic study of the fifth order integrable nonlinear evolution equations by using the tanh method," Applied Mathematics and Computation, vol. 174, no. 1, pp. 289299, 2006. 


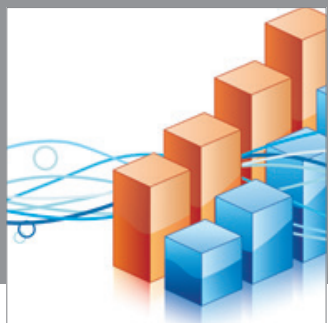

Advances in

Operations Research

mansans

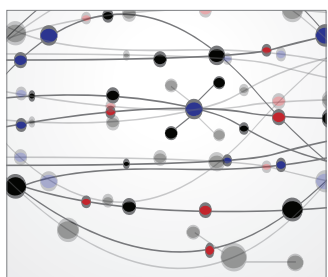

The Scientific World Journal
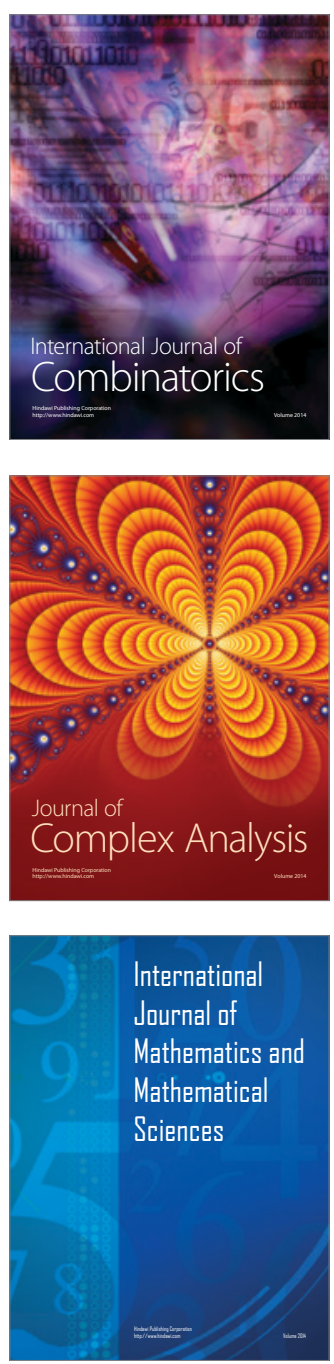
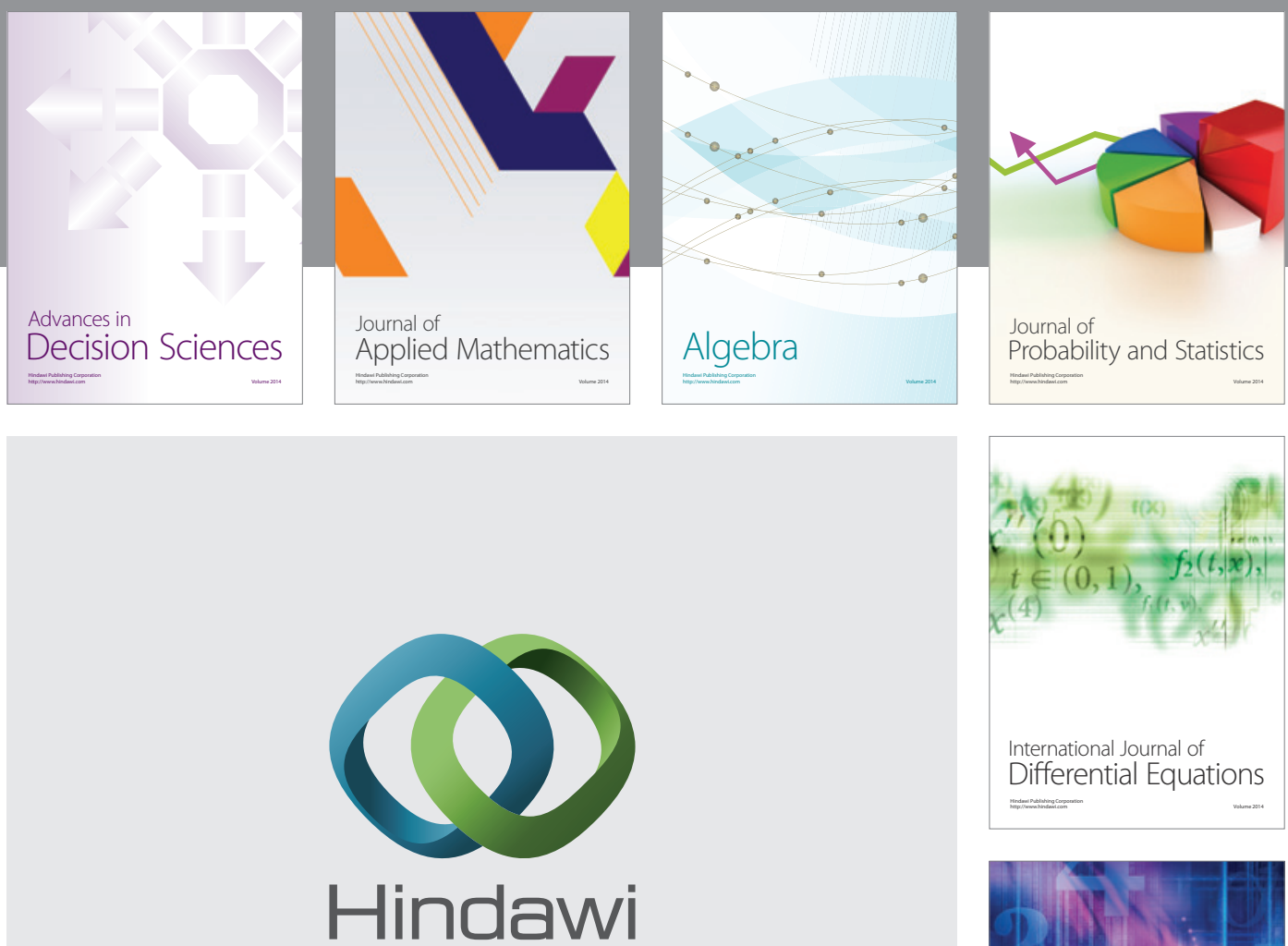

Submit your manuscripts at http://www.hindawi.com
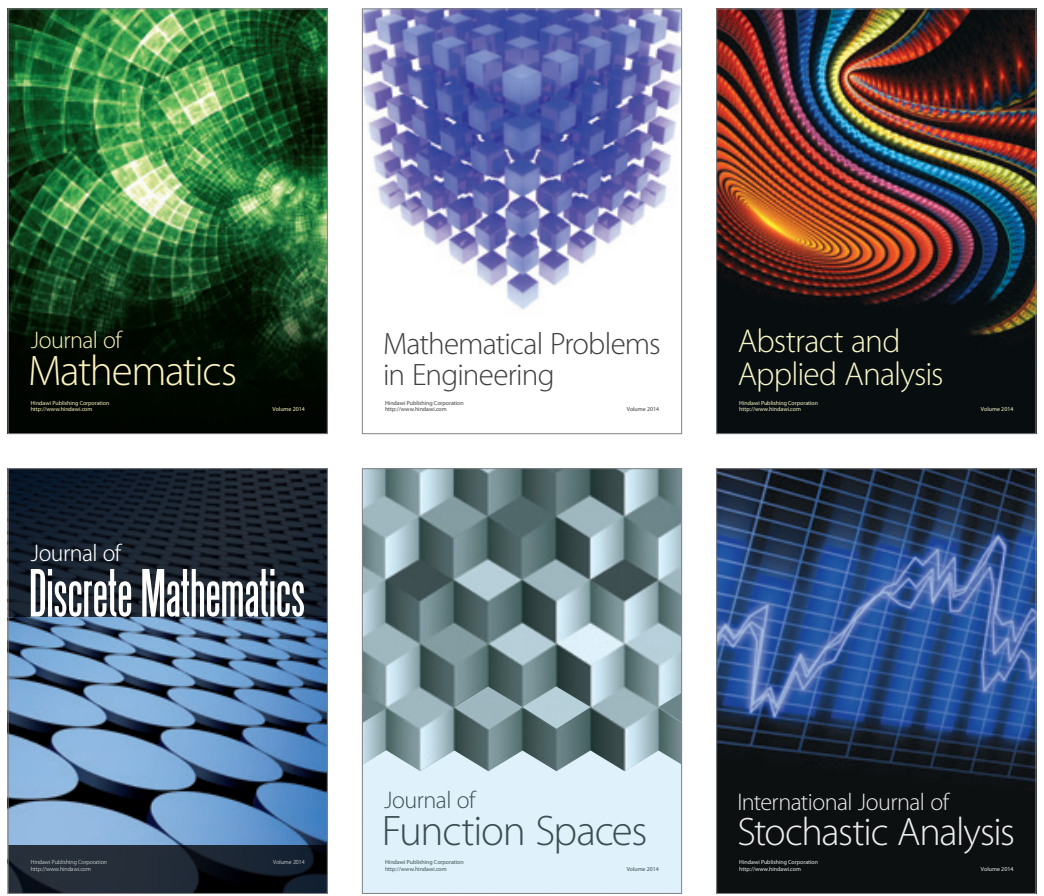

Journal of

Function Spaces

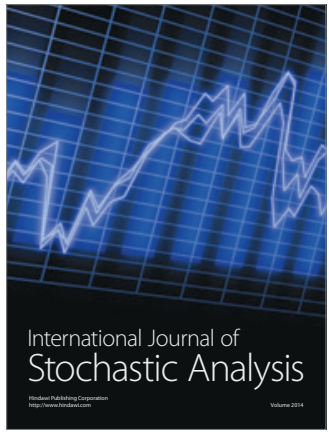

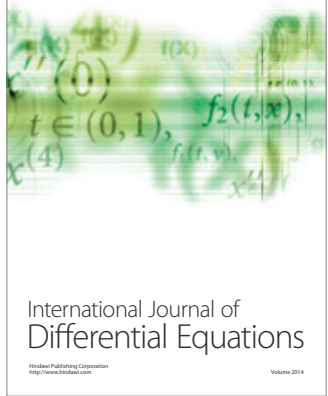
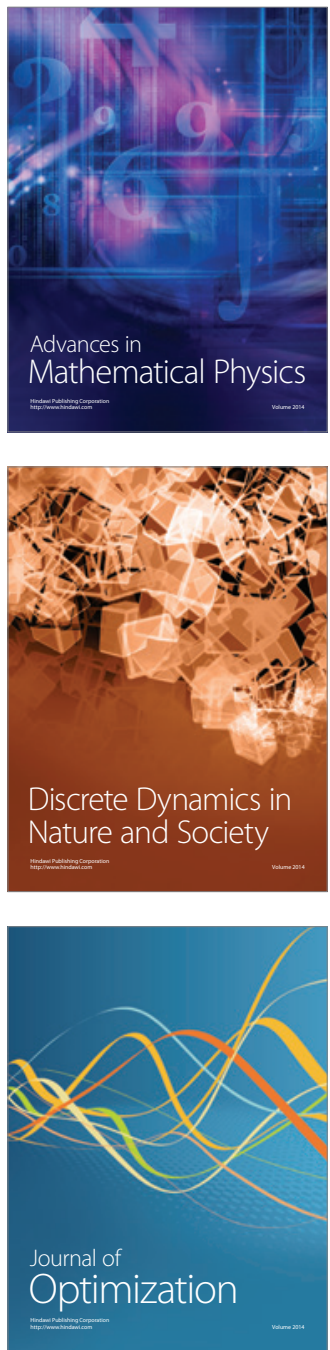